\title{
Self-consistent description of deformed nuclei at the proton drip line
}

\author{
Lidia S. Ferreira ${ }^{1}$, Enrico Maglione ${ }^{2}$ and Peter Ring ${ }^{3}$
}

${ }^{1}$ Centro de Física e Engenharia de Materiais CeFEMA and

Departmento de Física, Instituto Superior Técnico, Universidade de Lisboa, Av Rovisco Pais, 1049 001, Lisboa, Portugal

2 Dipartimento di Fisica e Astronomia "G. Galilei",

Via Marzolo 8, I-35131 Padova, Italy

and INFN, Sezione di Padova, Italy

${ }^{3}$ Physik-Department der Technischen Universität München, D-85748 Garching, Germany

\begin{abstract}
Proton radioactivity from deformed nuclei is described for the first time by a fully self-consistent calculation based on covariant relativistic density functionals derived from meson exchange and point coupling models.
\end{abstract}

\section{Introduction}

The production and detection of nuclei towards the limits of stability saw a considerable development in the last decade, due to a better capability to achieve fusion evaporation and multi fragmentation reactions [1] involving very small production cross sections, and producing exotic nuclei with extremely short half lives, below a microsecond. The position of the extremes of bound matter has been confirmed on the neutron side up to Magnesium, and on proton side for charges up to $\mathrm{Z}=83$, with only few exceptions of nuclei yet to be found. The observation of one proton radioactivity, mapped the drip-line between Tin and Bismuth. 
Nuclei at the proton drip-line, provide a challenging domain for spectroscopic studies far from stability [2]. Recent advances in tagging techniques lead to the discovery of many excited states [3], resulting in a significant progress on the knowledge of the nuclear structure properties of proton rich nuclei, and providing an important input towards the understanding of fundamental symmetries and of the nucleosynthesis of the elements. In fact, the path of the rapid proton capture reactions for the production of nuclei, can involve nuclei at the proton drip-line [4], but the reaction rates will depend strongly on details of the structure of nuclei along the path, and in particular, on the knowledge of resonances, since it is mainly dominated by resonant proton capture. Penning traps, have also allowed to isolate isomeric states, and to determine high precision mass measurements [5-7], that lead to a clear definition of proton separation energies, which are very import to establish the drip lines and for astrophysical calculations of the abundances.

In spite of the experimental advances in the area, a great part of the nuclear structure knowledge of nuclei at the limits of stability, still relies on theoretical extrapolations. In this context, the need to have a solid theoretical ground, with great independence from parameterizations, and with the capability to interpret the data and help the search of new emitters, is crucial.

Theoretical studies on proton radioactivity, were able to interpret the experimental half-lives and branching ratios measured in the observation of decay, and to predict the properties of the decaying state and shape of the emitter. Non-relativistic calculations using simple semi-classical WKB methods [8], were used for spherical emitters, while deformed proton emitters were studied more rigorously by identifying that the decay proceeds from a single-particle resonances $[9,10]$. Microscopic studies of deformed proton emitters allow to test the details of the nuclear wave function to which the half-lives are quite sensitive. The most consistent non-relativistic theoretical approach for proton emission is the non-adiabatic quasiparticle approach which is very successful [11] in bringing out several interesting features of deformed odd-even proton emitters including the triaxially deformed ones [12]. Its extension to odd-odd nuclei [13], suggested that it is possible to test the residual neutron proton interaction and to determine the neutron Nilsson level even if the neutron does not participate actively in the decay.

The above non relativistic microscopic models, rely on the various parameterizations of the nuclear mean field available in the literature, described in terms of phenomenological Wood-Saxon type forms, with parameters fitted to global nuclear properties, or by semiclassical considerations based on the liquid drop model and the proximity force. There is no interactions yet from first principles. 
Covariant density functional theory (CDFT) [14-16], might present some advantages. It is derived from relativistic quantum field theory, fulfils Lorentz invariance allowing to describe consistently the spin-orbit coupling, thus restricting the number of parameters of the functionals to be adjusted in order to reproduce bulk properties of spherical and deformed nuclei. The empirical pseudospin symmetry is also explained, and the model is consistent with the non linear realization of chiral symmetry. The functionals can be considered universal since they are valid all over the periodic table, where mean field theory is applicable.

A density dependence is introduced to allow for a quantitative description of the nuclear surface [17]. This can be achieved either by using nonlinear coupling terms or by considering coupling constants dependent on the density of the exchanged mesons. Examples of these interactions are the non-linear meson exchange model the NL3 [18] and the density dependent point coupling models the DD-PC1 [19], and the PC-PK1 [20], which were able to describe ground-state properties and collective excitations of $\beta$ stable and exotic nuclei at the extremes of stability [21]. Ground-state quadrupole deformations have been predicted for proton rich nuclei, and proton separation energies have been calculated, allowing the proton drip-line to be mapped with high precision $[21,22]$, and the single particle configurations and spectroscopic factors to be derived very successfully.

Relativistic mean-field calculations based on CDFT, have been used to interpret proton radioactivity from spherical nuclei [23, 24]. In our study of Ref. [23], we have performed a fully self-consistent relativistic mean field calculations for spherical nuclei, using the NL3 and DD-PC1 interactions. The decay process is described there within the exact formalism of quantum mechanics for decay of a resonance, and no additional parameters were introduced, besides the ones implicit in the definition of the NL3 and DD-PC1 interactions. The calculation was very successful in reproducing the experimental data, and provided a clear evidence for a mixing of configurations. The calculation of Ref. [24], interprets proton radioactivity, similarly to $\alpha$ decay, and not decay from a single particle resonance. The proton escape is simulated by a WKB tunneling process, where the potential barrier is extracted within the renormalization group approach. Therefore, it is not a self consistent calculation.

We have extended our spherical calculation in order to account for deformation [25], and in the present work, we discuss a fully self consistent calculation of proton emission from ${ }^{131} \mathrm{Eu}$, a well deformed nucleus. 


\subsection{Covariant density function theory for decay of deformed proton emitters}

In CDFT, the nucleons are described by the Dirac spinors $\psi$, interacting in an effective Lagrangian through the exchange of the isoscalar scalar $\sigma$, isoscalar vector $\omega$, and isovector vector $\rho$ mesons, and the electromagnetic field. This simple model, with interaction terms linear in the meson fields, does, however, not provide a quantitative description of the nuclear surface properties, thus the need for an effective density dependence, as mentioned above. From the Lagrangian density the classical variation principle leads to the equations of motion, which are the Dirac equation for the nucleons, equivalent to the Kohn-Sham equations in non-relativistic density functional theory, and the Klein-Gordon equations for the meson fields. Pairing correlations are taken into account in the constant gap approximation. This set of equations is non-linear and is solved by iteration starting with an initial guess for the potentials, until self-consistency is achieved, and the final mean field interaction obtained.

For the calculation of proton emission from ${ }^{131} \mathrm{Eu}$, we have used the relativistic point-coupling models, DD-PC1 and PC-PK1 of the Peking group, both successful in describing nuclear properties, but with some differences between them [26,27], regarding their structure and fitting procedure. The functional PC-PK1 has phenomenological parameters fitted to the binding energies and radii of a large number of spherical semi-magic nuclei all over the periodic table. The functional DD-PC1, was instead fitted to masses of 64 heavy deformed nuclei and a few nuclear matter properties. Therefore, both interactions describe spherical and deformed nuclei differently, being the masses of deformed nuclei better reproduced by the DD-PC1, whereas for spherical nuclei, the masses are over-bound.

The scalar and vector potentials that describe the deformed proton emitter, were obtained in a fully self-consistent way, by solving the relativistic mean field equations for the even-even daughter nucleus including pairing correlations in the BCS approximation. The resulting self-consistent axial potentials $S(\mathbf{r})$ and $V(\mathbf{r})$ were then expanded in spherical harmonics with different $L$-values, obtaining the corresponding potentials $S_{L}(r)$ and $V_{L}(r)$ on a fine mesh in r-space. The latter interactions, were then used to calculate the proton Nilsson resonances, which are the solutions of the Dirac equations imposing outgoing wave boundary conditions. This was achieved by solving a full coupled channel problem in coordinate space, leading to a precise description of the energy, width and wave function of the proton resonances. 


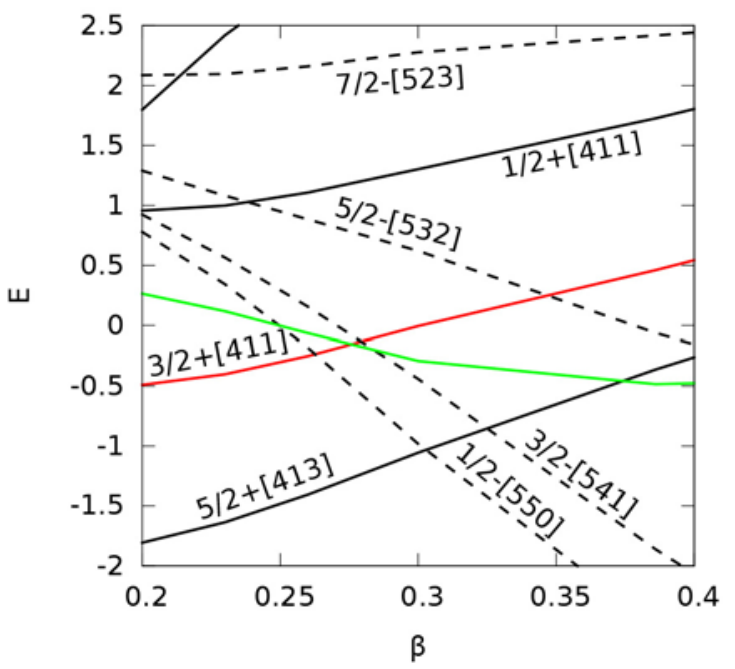

Figure 1: Proton Nilsson resonances in ${ }^{131} \mathrm{Eu}$ as a function of quadrupole deformation $\left(\beta_{2}\right)$ using the DD-PC1 field. The Fermi energy is represented by the green line.

This is a complex problem, solved previously only for $\mathrm{K}=1 / 2^{+}$resonant states by the Beijing group [28], and in [29] as an extension of the complex scaling method. We have solved the full relativistic coupled channel problem for any value of the angular momentum in coordinate space [25], using similar techniques as in the non-relativistic case [30]. The Nilsson diagram for resonances in ${ }^{131} \mathrm{Eu}$ is presented in Fig. 1 for the self-consistent density dependent point coupling DD-PC1 interaction. Radioactive decay of ${ }^{131} \mathrm{Eu}$, was observed from the ground state to the ground and first excited $2^{+}$states of the daughter nucleus ${ }^{130} \mathrm{Sm}[31,32]$. The nucleus is highly deformed, and if there is no change of deformation during the decay, ${ }^{131} \mathrm{Eu}$, should have a similar deformation to the one expected for ${ }^{130} \mathrm{Sm}$, i.e. a value $\beta_{2} \approx 0.34$, deduced from the empirical relation of Ref. [33]. As it can be seen from Fig.1, the $3 / 2^{+}[411]$ state is close to the Fermi surface for a large quadrupole deformation, so it is the candidate to be the decaying state, similarly to what was predicted by the non-relativistic calculation [34].

We have calculated the half-life for decay from the various states close to the Fermi surface and the branching ratios for the decay of these states, to the first excited $2^{+}$state of the daughter ${ }^{130} \mathrm{Sm}$. For details, see Ref. [25].

The half-life depends strongly on the quantum numbers of the decaying state, and also on details of the interaction like for example the nuclear radius, and strength of the spin-orbit force. In contrast, the branching ratios 
Table 1: Branching ratio for decay from different $\mathrm{J}^{+}$states of ${ }^{131} \mathrm{Eu}$ to the first excited $2^{+}$state of the daughter ${ }^{130} \mathrm{Sm}$, with a deformation $\beta_{2}=0.34$, and for the DD-PC1 model. The experimental branching ratio is $0.24(5)$ [31].

\begin{tabular}{ll}
\hline \hline$J^{\pi}$ & Branching \\
\hline $3 / 2^{+}[411]$ & 0.191 \\
$1 / 2^{+}[411]$ & 0.024 \\
$5 / 2^{+}[413]$ & 0.031 \\
$5 / 2^{+}[402]$ & 0.023 \\
$7 / 2^{+}[404]$ & 0.029 \\
\hline \hline
\end{tabular}

are quite stable regarding these quantities, but are very sensitive instead to details of the wave function components with different angular momentum, since their calculation involves the sum over all allowed partial decay widths for decay, divided by the total width, so they are a ratio between very different amplitudes. Therefore, the branching ratio depends on a balance between details of the wave function components, and for this reason an excellent probe of nuclear structure properties.

We present in Table 1 the calculation, of the branching ratio for decay from the Nilsson single particle states of ${ }^{131} \mathrm{Eu}$, close to the Fermi energy, to the $2^{+}$state of the daughter nucleus, assuming they have a quadrupole deformation predicted by the Grodzins relation, $\beta_{2}=0.34$. As it can be seen, only the $3 / 2^{+}[411]$ state interprets perfectly the experimental value of $0.24(5)$ [31], in agreement with what was found in the non-relativistic calculation [34], and also the predictions based on microscopic-macroscopic mass calculations [35]. The calculation was done with the DD-PC1 model, but similar conclusions can be drawn if the PC-PK1 is used, since only minor differences in the behaviour of the half-lives was found.

This is the first fully self-consistent calculation of proton emission from deformed nuclei, with interactions derived from covariant density functionals. It was very successful to reproduced the experimental data, and has provided a new test of the relativistic point-coupling functionals, the DDPC1 and the PC-PK1.

\section{Conclusions}

In conclusion, theoretical calculations within relativistic mean field models present some advantages with respect to the non-relativistic ones, concerning 
the parameterization of the nuclear interaction, but a unique parameterization for the Lagrangian, which is able to describe properties of nuclei from light to very heavy, and from the proton drip-line to the neutron one, is still not available. Therefore, it is desirable to provide a new test of the interactions derived from the relativistic density functionals, and observe their performance. Proton radioactivity provides this possibility. We have presented the first self-consistent calculation in the literature for decay of deformed emitters, using interactions derived from covariant density functional theory, and determined exactly the Nilsson resonances and the decay observables. We have used two density dependent point coupling models, the DD-PC1 [19] and the PC-PK1 [20], and concluded that they could also describe proton radioactive nuclei, and predict their properties.

From the above discussion, one can conclude that a very solid theory exists that can describe proton radioactivity, interpret the data, and predict features of the structure of nuclei at the extremes of stability, within covariant relativistic mean field theory.

\section{References}

[1] M. Thoennessen, AIP Conf.Proc. 802 (2005) 250.

[2] B. Blank and M. J. G. Borge, Prog. Part. Nucl. Phys. 60 (2008) 403.

[3] P.T. Wady, et al. Phys. Lett. B 740 (2015) 243.

[4] M. Arnould, S. Goriely, Phys. Reports 384 (2003) 1.

[5] C. Rauth et al., Phys. Rev. Lett. 100 (2008) 012501.

[6] H. J. Kluge, Int. Journal of Mass Spectrometry 349 (2013) 26.

[7] M. Kankainen, et al., Proceedings of Science, NIC XII 029 (2013).

[8] S.Åberg, P. B. Semmes, W. Nazarewicz, Phys. Rev. C 56 (1997) 1762.

[9] L. S. Ferreira, E. Maglione, Phys. Rev. Lett. 86 (2001) 1721.

[10] C. N. Davids and H. Esbensen, Phys. Rev. C 69 (2004) 034314.

[11] G. Fiorin, E. Maglione, L. S. Ferreira, Phys. Rev. C 67 (2003) 054302.

[12] P. Arumugam, L. S. Ferreira, E. Maglione, Phys. Lett. B680 (2009) 443. 
[13] M. Patial, P. Arumugam, L. S. Ferreira, E. Maglione, Phys. Rev. C80 (2013) 004300.

[14] M. H. Johnson and E. Teller, Phys. Rev. 98 (1955) 783.

[15] H.-P. Dürr, Phys. Rev. 103 (1956) 469.

[16] J. D. Walecka, Ann. Phys. (N.Y.) 83 (1974) 491.

[17] J. Boguta and A. R. Bodmer, Nucl. Phys. A 292 (1977) 413.

[18] G. A. Lalazissis, J. König, P. Ring, Phys. Rev. C55 (1997) 540.

[19] T. Nikšić, D. Vretenar, P. Ring, Phys. Rev.C78 (2008) 034318.

[20] P. W. Zhao, et al, Phys. Rev. C82 (2010) 054319.

[21] G. A. Lalazissis, et al. Nucl. Phys. A 650 (1999) 133.

[22] G. A. Lalazissis, D. Vretenar, P. Ring, Phys. Rev. C69 (2004) 017301.

[23] L. S. Ferreira, E. Maglione, P. Ring, Phys. Lett. B701 (2011) 508.

[24] Q. Zhao, et al, Phys.Rev. C90 (2014) 054326.

[25] L. S. Ferreira, E. Maglione, P. Ring, Phys. Lett. B, in press.

[26] S. E. Agbemava, et al, Phys. Rev. C89 (2014) 054320.

[27] Q. S. Zhang, et al, Front. Phys. 9 (2014) 529.

[28] Z. P. Li, et al, Phys. Rev. C81 (2010) 034311.

[29] M. Shi, et al, Phys. Rev. C90 (2014) 034319.

[30] L. S. Ferreira, E. Maglione, R. Liotta, Phys. Rev. Lett. 78 (1997) 1640.

[31] A. A. Sonzogni, et al, Phys. Rev. Lett. 83(1999) 1116.

[32] C. N. Davids, et al. Phys. Rev. Lett. 80 (1998) 1849.

[33] L. Grodzins, Phys. Lett. 2 (1962) 88.

[34] E. Maglione, L. S. Ferreira, Phys. Rev. C61(2000) 047307.

[35] P. Möller, et al, At. Data Nucl. Data Tables 66 (1997) 131. 